\title{
Transmittance measurement of the in vivo human eye with a double-pass system
}

\author{
Roberto SÁNCHEZ ${ }^{1 *}$, ANíbal DE PAUL ${ }^{1}$, Francisco BURGOS-FERNÁNDEZ ${ }^{2}$, \\ MeritXell VILASECA ${ }^{2}$, JAUMe PUJOL $^{2}$, LuIS ISSOLIO ${ }^{1,3}$ \\ ${ }^{1}$ Instituto de Investigación en Luz, Ambiente y Visión, \\ Avenida Independencia 1800, T4002BLR Tucumán, Argentina \\ ${ }^{2}$ Centre de Desenvolupament de Sensors, Instrumentació i Sistemes, Universitat Politecnica de Catalunya, \\ Rambla de Sant Nebridi 10, 08222 Barcelona, España \\ ${ }^{3}$ Universidad Nacional de Tucumán, \\ Avenida Independencia 1800, T4002BLR Tucumán, Argentina \\ ${ }^{*}$ Corresponding author: rsanchez@herrera.unt.edu.ar
}

Purpose: To develop a methodology based on a double-pass system to obtain information about the transmittance of ocular media. Methods: The procedure consists of recording double-pass images at different powers of a laser diode of $780 \mathrm{~nm}$ and determining the scattering in an area between 25-35 arcmin of each image. The scattering showed linear behavior in respect to the irradiance of the laser, and the slope of the linear fit was proportional to the transmittance squared of the media evaluated. An artificial eye with different filters was tested first. Then, fifteen subjects with clear ocular media were divided into two groups: ten subjects classified by the iris color were recruited for the measurements of an ocular transmittance index and the estimation of the transmittance (group A), and another five subjects were selected for measurements with neutral filters (group B). Results: The measurements performed in group A presented a mean transmittance of $42.95 \%$. No differences in the transmittance were found between subjects with different iris color $(p=0.154)$. Measurements in group B showed a good correlation $(r=0.959, p<0.001)$ between the expected and the measured value for the transmittance. Conclusion: We proposed and evaluated a method to determine the transmittance of the eye in vivo using the double-pass system.

Keywords: double-pass, transmittance, human eye.

\section{Introduction}

Knowledge of transmittance of the eye can be useful for both clinical applications: where it is relevant to estimate the amount of light actually reaching the photoreceptors, and lighting research, where it is often necessary to characterize visual stimuli with 
both luminance and retinal illuminance, which includes in its rigorous definition the transmittance of the eye [1].

BOETTNER and Wolter, as well as GEERAETS and BERRY, have measured the direct and total transmittance of isolated components of the ocular media, including the cornea, aqueous, lens and vitreous, but without including retina; using enucleated eyes of human donors and rhesus monkeys [2- $\underline{4}]$. They considered a wide range of wavelengths including visible, near-ultraviolet (UV) and near-infrared. Those results were confirmed by ALPERN et al. through in vivo measurements performed on subjects with structural abnormalities in the fundus of the eye (chorioretinal coloboma). That condition allowed the determination of the amount of light transmitted by the eye, through a direct comparison of the component reflected in the sclera of a light beam with a reference [ [ $]$ ]. Dillon et al. proposed an invasive method for measuring the absorption spectrum of the anterior segment of the intact eye. The true spectrum of light that is transmitted to the retina was calculated for four different species that are commonly used in animal model experiments [6]. VAN NORREN and Vos have estimated the spectral transmittance of the eye as the measured difference in the scotopic visual sensitivity of two wavelengths with the same rhodopsin absorbance [7]. Many other studies of the human ocular media were limited to a single component of the eye, usually the cornea or lens. In 2007, VAN DE KRAATS and VAN NORREN proposed empirical equations to estimate changes in ocular transmittance as a function of the eye age []ㅡ. Based on existing data and these equations, the International Commission on Illumination (CIE) established the transmittance of the standard observer for UV, visible and infrared which can be used as a reference [9]. Currently, there is no simple procedure for measuring the transmittance of the whole eye of a healthy subject.

The purpose of this work was to take advantage of the double-pass (DP) technique to estimate in a direct way the transmittance of the eye at a given wavelength. The DP method is a simple, fast, safe, and non-invasive technique to obtain information about the energy that enters the eye, crosses the ocular media, reflects in the fundus and returns. This technique is based on imaging a point source on the retina, and then recording the reflected light through a CCD camera [10]. The acquired image is the autocorrelation of the point spread function (PSF) of the eye [11], which describes how the optical system behaves against a point light source [12].

It is known that in the retina, light is reflected by different layers and a significant portion of the light reflected by the background comes from the choroid and is dependent on pigmentation and wavelength $[\underline{13}, 14]$. In a DP system, the imaging formation approximates that of a confocal system, so that most of the light coming from the deeper layers not conjugated to the camera are spreading, adding a background signal to the recorded image. Thus, the DP image shows a very narrow peak mounted on a fairly wide tail [15]. Scattering affects both the peak and the wide-angle part of the PSF, unlike aberrations, which are limited to the very narrow-angle part of the PSF [ $\underline{16}-\underline{18}]$. Several authors showed that it is possible to obtain information about intraocular scattering by analyzing the peripheral zone of the DP image up to 20 minutes of arc [19-21]. 
Our goal was to develop a non-invasive methodology based on a DP system to measure the transmittance of the ocular media. In this work, we propose a simple method to determine the direct transmittance of the eye including the retina, based on the acquisition and analysis of DP images.

\section{Methods}

To analyze the performance of the method proposed in human eyes, we conducted a study in a group of fifteen healthy volunteers aged between 25 and 45 of the Universidad Nacional de Tucumán. The experimental design was carried out in two stages, for which the subjects were randomly divided into two groups. Ten subjects were recruited for a first experiment (group A), in which an ocular transmittance index (OTI) was measured and an estimation of the transmittance of the ocular media was performed. Later, in a second experiment, where the range of low transmittance was measured to test the additivity, five different subjects were recruited (group B). The inclusion criteria were as follows: corrected visual acuity of 20/20 or higher and the absence of a history of ocular diseases, surgery or pharmacological treatment. In addition, only individuals with spherical refraction in the range of -1.5 to $+1.5 \mathrm{D}$, and astigmatism less than $-1.0 \mathrm{D}$ have been included. In each case, the dominant eye was chosen to perform the measurement.

The subjects of the group A were classified as dark-eyed (four dark brown and three light brown) and three as light-eyed (blue), according to a classification by simple iris observation [22]. Measurements of the OTI index were performed in the five subjects of the group B with and without neutral density filters (ND 0.01, ND 0.1, and ND 0.3).

Ethical approval for this study was obtained from the Comité de Ética en Investigación (CEI) of the Universidad Nacional de Tucumán and Consejo Nacional de Investigaciones Científicas y Técnicas (RESOLUCION N²6/2018). All the subjects were informed about the object of the study, and a written informed consent was obtained, following the tenets of the Declaration of Helsinki.

\subsection{Experimental setup and image acquisition}

A scheme of the optical system used in this study is shown in Fig. 1. A point source (O) from a $780 \mathrm{~nm}$ laser diode (MC7850CPWR-SMF) coupled by a single-mode optical fiber to a collimator lens $\mathrm{L} 1(f=15 \mathrm{~mm})$ is projected onto the retina $\left(\mathrm{O}^{\prime}\right)$. After back reflection on the retina and a double pass through the ocular media, a CCD camera records the double-pass image $\left(\mathrm{O}^{\prime \prime}\right)$. The diaphragm $\mathrm{PE}(\varnothing=2 \mathrm{~mm})$ is conjugated to the pupil plane of the eye and acts as the effective input pupil of the system. After being reflected by a beam splitter BS1 and mirror M1, the beam passes through the Badal system formed by the lenses L 2 and $\mathrm{L} 3(f=200 \mathrm{~mm})$ and by the mirrors M2 and M3 that control the vergence of the beam, allowing correction of refractive errors such as myopia or hyperopia of the eye before performing the measurement. After reflection in the mirror M4, the eye forms the image of the point source on the retina. A second diaphragm PS ( $\varnothing=4 \mathrm{~mm})$, located behind the beam splitter (BS2), is also conjugated 


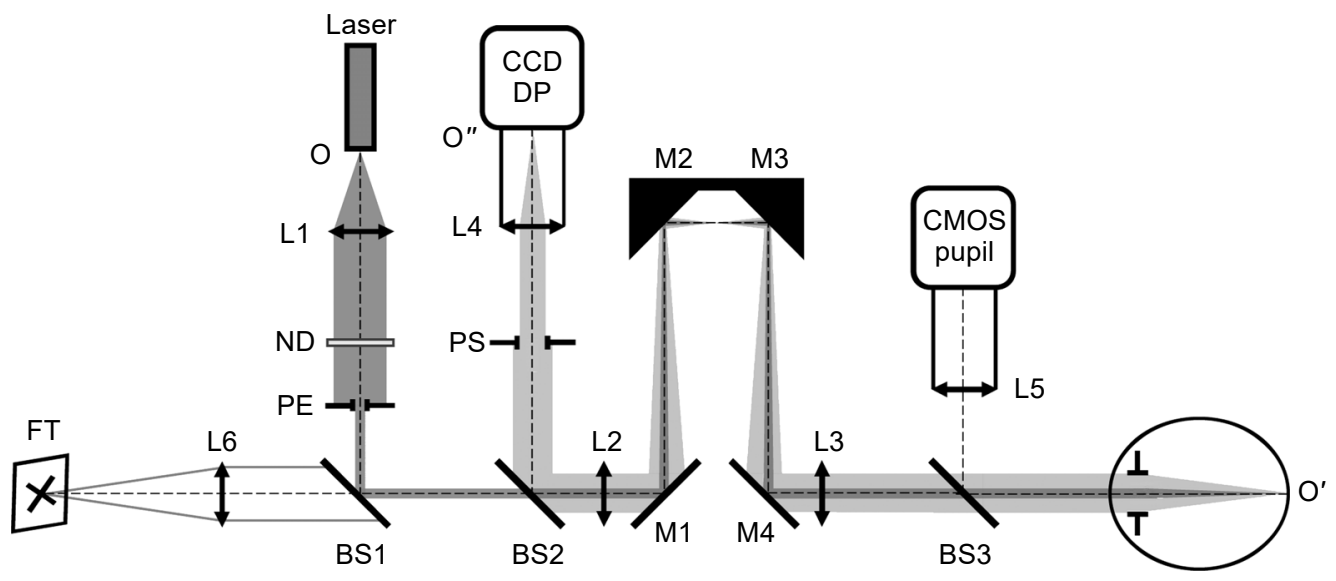

Fig. 1. Schematic representation of the double-pass setup used for the purpose of this work. See the text for further details.

to the pupil of the eye and acts as the effective exit pupil (provided that the natural pupil of the eye is greater than PS). Then a lens L4 $(f=100 \mathrm{~mm})$ forms the double -pass (DP) retinal image in a CCD camera (UI-2220ME-M, 8 bits, $768 \times 576$ pixels) integrating light from the retina during the set exposure time. The subject's head was placed in a chin rest which allows the proper centration and control of natural pupil with respect to the artificial one. A camera CMOS (UI-1221LE-M-GL, 8 bits, $752 \times 480$ pixels) and the lens L5 ( $f=50 \mathrm{~mm})$ were mounted to ease this process. The fixation of the eye was facilitated using a fixation target (FT) located at the optical infinity by the lens L6 $(f=35 \mathrm{~mm})$.

To eliminate back reflections from lenses L2 and L3, these elements were tilted slightly, so that the specular reflection at the interfaces of these lenses was deflected off the field picked up by the CCD sensor. The corneal reflex and the lens reflex (Purkinje images) were not observed in the DP image captured, due to a small decentering of the measuring beam with respect to the pupil of the subject. Other diffuse reflections were reduced by light traps (not shown in Fig. 1). Finally, there were still spurious reflexes that were integrated by the sensor during the recording of the images. However, these were considered during postprocessing by subtracting the captured background when the eye was removed.

For all the measurements, the exposure levels were never greater than the maximum permissible exposure $\left(14.45 \mathrm{~W} / \mathrm{m}^{2}\right)$ which was established by the current standard regulating the use of laser radiation in living tissue [23].

The laser diode, cameras CCD and CMOS, and the movement of the Badal by means of a stepper motor were controlled using a personal computer through a customized software developed in Visual Studio, while C\#, EMGU CV, and a MATLAB script were used for image processing.

The measurements were taken in a dark room with the lights of the lab turned off after the subject was adapted to the darkness with the purpose of obtaining the largest 
possible natural pupil, while the exit pupil of the system was controlled by the artificial diaphragm (PS). In each record, six DP images and the corresponding background were taken per subject (each obtained with an exposure time of $250 \mathrm{~ms}$ ), after correcting spherical refractive errors with the Badal of the instrument. No glasses or contact lenses were used for the optical correction during measurements. From each set of six images, an average image was calculated and then the background was subtracted from this average. Finally, a cropped version of this DP image $(256 \times 256$ pixels $)$ was used for the analysis.

\subsection{Methodological proposal}

As pointed out by several authors $[\underline{19}, \underline{24}]$, the double-pass (DP) image contains the effects of both aberrations and scattering. When the lower-order aberrations are corrected, the DP PSF in a normal eye without corneal ectasia or other cause for irregular astigmatism is affected predominantly by higher-order aberrations and light scatter. While the aberrations influence the light distribution at the central region of the DP image, the intraocular scattering causes an increment of the intensity further away from the central maximum of the image. Therefore, in this study we decided to analyze a peripheral portion of the DP PSF. The region of interest (ROI) chosen for the analysis was a ring between 25 to 35 minutes of arc, whose center was in the centroid of the spot of the cropped DP image (Fig. 2). Using the algorithm described by HofER et al. [25], the centroid location of the DP spot was iteratively evaluated in windows of decreasing size, each centered on the previous estimate.

To determine the transmittance of the ocular media, a series of DP images were recorded, each one was taken at different intensity of the laser diode, which was con-

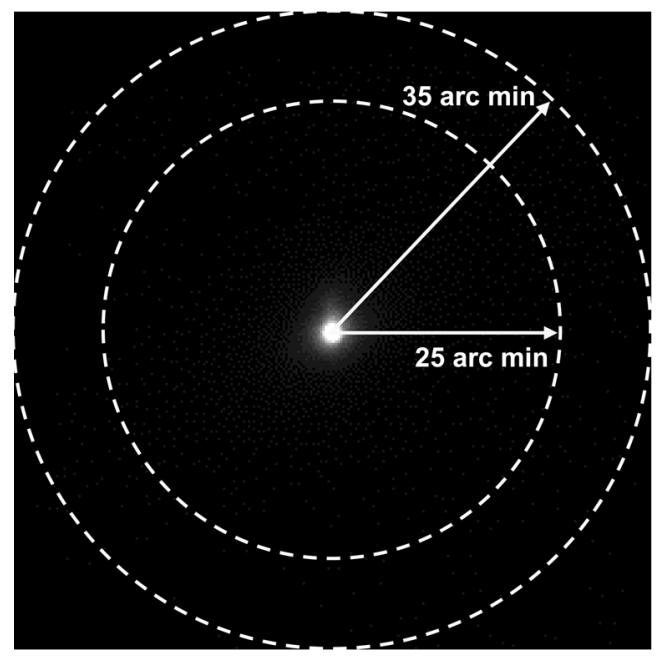

Fig. 2. Definition of the region of interest in the double-pass (DP) image. DP image with two circles in dashed line, the inner corresponding to 25 minutes of arc, and the outer corresponding to 35 minutes of arc. The ROI evaluated was the area between both circles. 
trolled by the power supply of the laser. The irradiance in the pupil plane was measured with a detector (E2V, Spindler \& Hoyer). The gray level in the ROI increased as the intensity of the laser increased. The average of the scattering computed as a mean gray level (which we will call hereafter double-pass scattering (DPS)) varies linearly with the irradiance in the pupil plane. We have used the slope of the DPS/irradiance line to derive an ocular transmittance index (OTI). As the light had to cross the ocular media twice to reach the CCD sensor, it was expected that the OTI slope has a functional relationship with the transmittance $T$, as OTI $\propto T^{2}$.

\subsection{Measurements in an artificial eye with filters}

To test the proposed methodology, measurements were made with an artificial eye built in the laboratory consisting of two lenses simulating the cornea $(f=25 \mathrm{~mm})$ and the lens $(f=50 \mathrm{~mm})$, plus a black diffuser screen (black cardboard) located in the focal plane of the lens system acting as a reflective retina. In order to simulate eyes with several transmittances, neutral filters of different optical density (ND 0.1, ND 0.3, and ND 0.5) were placed in front of the artificial eye. Additionally, measurements with photography effect filters - Black Pro-Mist (BPM) 1 and 2 from Tiffen, and Cinegel R3020 (from Rosco) - that have been shown to be very suitable to simulate the same type of scattering produced by an eye with cataracts were performed [26, 27].

The available information provided by the filters manufacturer was limited to the range of 350 to $700 \mathrm{~nm}$, therefore the transmittance of all filters used in this work was measured at $780 \mathrm{~nm}$, and those with transmittance values comparable to real eyes were selected. The transmittance measurements were performed using the collimated laser diode as an illuminator and a detector (E2V, Spindler \& Hoyer, photodiode type S2386-5K) to record the irradiance received. The arrangement shown in Fig. 3 was used to measure the transmittance in the filters.

The transmittance of each filter is given as quotient $E_{\mathrm{d}} / E_{\mathrm{f}}$, where $E_{\mathrm{d}}$ and $E_{\mathrm{f}}$ denote the irradiances with no sample present and after passing through the sample, respectively. The photodiode of the sensor measures only the direct radiation behind the filter. This component is also called the focusable transmittance and is important for understanding the deposition of laser energy on the retina.

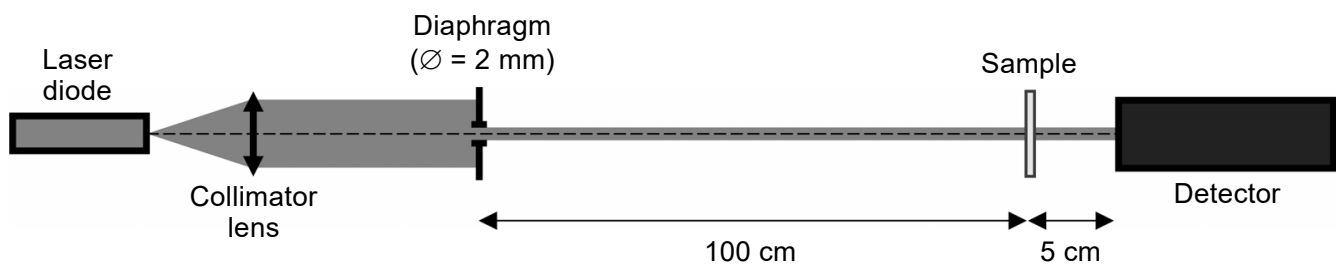

Fig. 3. Schematic representation of the setup used for transmittance measurements in the filters. The laser diode was the same used in the double-pass system described previously. The detector implemented was E2V (Spindler \& Hoyer). The E2V is an active detector based on the photodiode S2386-5K, with a sensitive area of $2.4 \times 2.4 \mathrm{~mm}^{2}$, a spectral range of 320 to $1100 \mathrm{~nm}$ and an internal amplification of 11 . 


\subsection{Impact of reflectance on ocular transmittance index}

The energy in the DP image also depends on the fundus reflectance of the human eye or the reflective surface of the artificial eye. To analyze the effect of this factor on the measurement, DP images of the artificial eye with different reflective surfaces (white cardboard and black cardboard) were taken and the OTI index was obtained. The reflectance of the cardboards for $780 \mathrm{~nm}$ was measured using a spectroradiometer (SpectraScan PR-715, Photo Research).

In order to analyze the OTI independence regarding the variation of the reflectance between different retinas, three subjects of group A and two subjects of group B were evaluated. These subjects were chosen because in a pilot experiment they presented very similar OTI values. The set of DP images were obtained considering the same intensity of the laser.

\subsection{Impact of defocus on ocular transmittance index}

The DP technique provides combined information of both aberrations and small-angle intraocular scatter. These two factors influence the light distribution on the retina. LOGEAN et al. [28] found that the width of the DP PSF after adaptive optics correction of the ocular aberrations is always smaller than the width of the DP PSF obtained in the same conditions but without the correction, indicating a decrease in the optical quality of the DP image due to the effect of high-order aberrations. Regarding low-order aberrations, ARTAL et al. [19] measured the effect of induced defocus on the DP PSF to obtain an acceptable range of uncorrected refractive errors (defocus and astigmatism) during the calculation of an objective scatter index. To asses this issue, we measured OTI in the artificial eye for different amounts of defocus from $-1.0 \mathrm{D}$ to $+1.0 \mathrm{D}$, in steps of $0.25 \mathrm{D}$, induced by means of the Badal system available in the experimental setup.

\subsection{Statistical analysis}

The Shapiro-Wilk test was used to evaluate the normal distribution of the DPS, OTI, and transmittance values. Since the OTI data did not meet the criteria for normal distribution, the Mann-Whitney $U$-test was used to compare the data between different conditions considered in group A (dark eyes, light eyes), between the OTI for the two cardboards (black, white), between the DPS of the five subjects evaluated at same laser power, between the OTI for different induced defocuses. The coefficient of determination $\left(R^{2}\right)$ was calculated to assess the relationships between the DPS, the OTI, and the transmittance. A value of $p<0.05$ was considered statistically significant.

An error propagation analysis was carried out taking into account the sensitivity of the CCD sensor, the error made in averaging the gray levels in the region of interest, the errors made during the transmittance measurements of the filters, and the errors made during the data fitting trough least squares to obtain both OTI and the final value of the transmittance. 


\section{Results}

The measured transmittances at $780 \mathrm{~nm}$ in each of the filters used with the artificial eye are shown in Table 1.

Figure 4 shows a sequence of double-pass (DP) images, taken at four different powers of the laser source, with the irradiance value for each case.

Figure 5a shows the radial profiles in the logarithmic scale obtained from the DP images (averaged of pixels at different radii) taken in the artificial eye without any filter.

$\mathrm{T}$ a b l e 1. Direct transmittance measurements.

\begin{tabular}{ll}
\hline Filter & $\begin{array}{l}\text { Measured } \\
\text { transmittance [\%] }\end{array}$ \\
\hline ND 0.01 & $97.0 \pm 3.5$ \\
ND 0.1 & $87.7 \pm 2.3$ \\
ND 0.3 & $56.2 \pm 1.7$ \\
ND 0.5 & $34.1 \pm 1.6$ \\
BPM1 & $68.0 \pm 0.8$ \\
BPM2 & $58.5 \pm 0.6$ \\
R3020 & $84.6 \pm 1.2$ \\
\hline
\end{tabular}
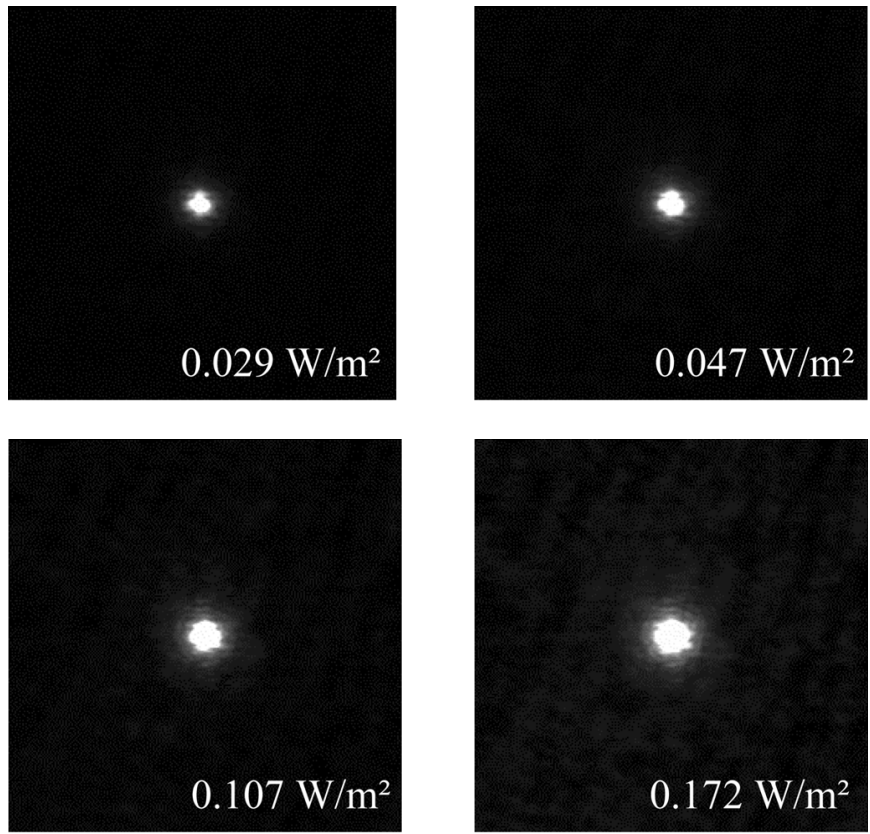

Fig. 4. Example of double-pass (DP) images recorded at four different powers of the laser. The DP images correspond to measurements made in the artificial eye. The corresponding value of the laser irradiance measured in the pupil plane is shown in the lower right corner. 


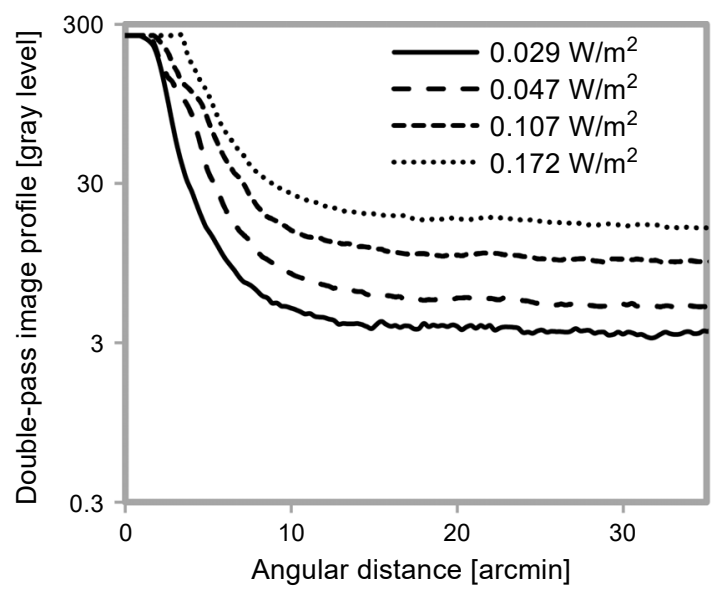

a

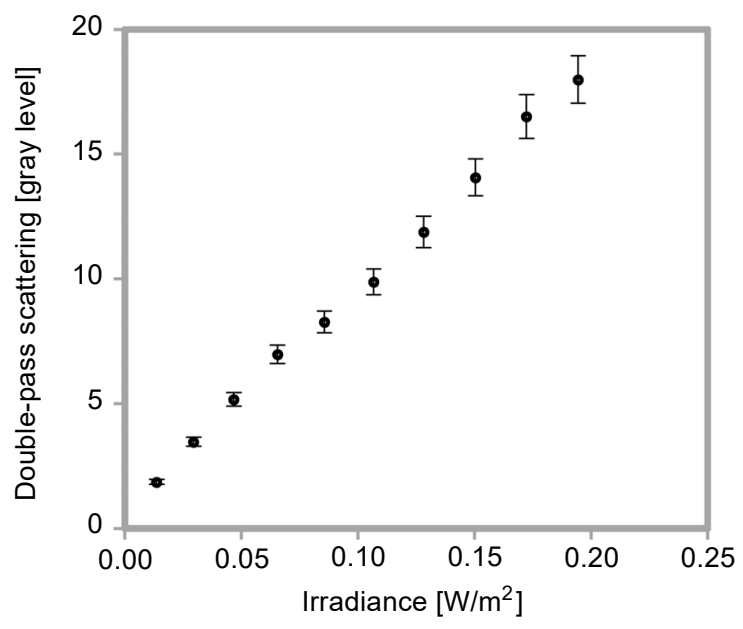

b

Fig. 5. Example of double-pass (DP) image profiles obtained in the artificial eye. (a) The curves correspond to four different intensities of the laser diode $\left(0.029,0.047,0.107\right.$, and $\left.0.172 \mathrm{~W} / \mathrm{m}^{2}\right)$. (b) Double -pass scattering (DPS) as a function of the irradiance in the pupil plane. DPS was computed as a mean of the gray levels in a ring of 25 to 35 minutes of arc of the DP image. The error bars correspond to the uncertainty $(5.4 \%$ of the measured value), obtained from the propagation of the error.

As the power of the laser increases, the central area of the curve saturates and a measurable variation in the peripheral zone (the skirt of the curve) can be observed. Double -pass scattering (DPS) calculated as the average gray level in the ROI as a function of the irradiance of the laser in the pupil plane is shown in Fig. $5 \mathbf{b}$.

In Fig. 6a are plotted the DPS values as a function of the irradiance of the laser in the pupil plane for each of the considered filters, as well as for the artificial eye without a filter. For each condition, a straight line was fitted to the data through least squares and the slopes OTI were obtained. The higher the transmittance of the filter, the higher the OTI value (Fig. 6b). 

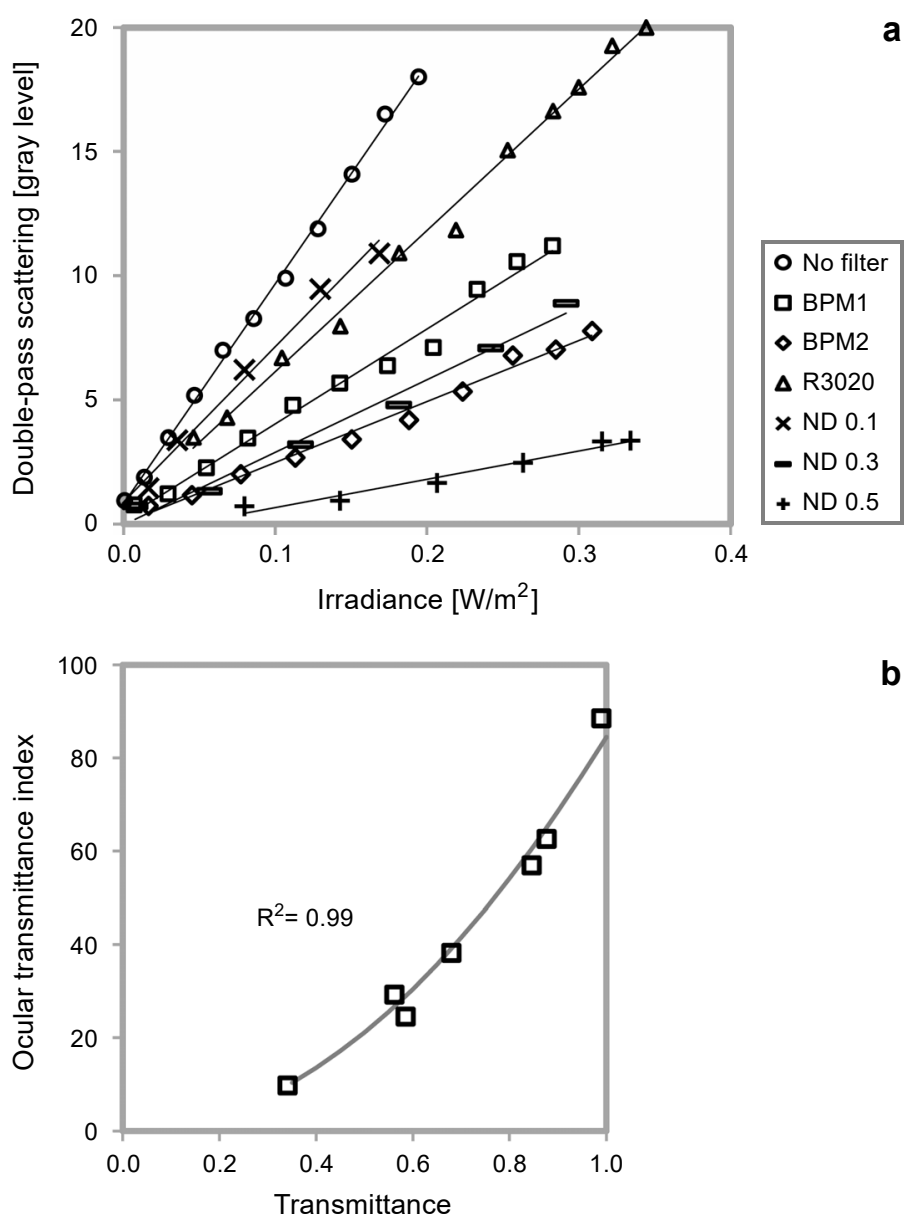

b

Fig. 6. Double-pass scattering (DPS) as a function of the irradiance in the pupil plane obtained in the artificial eye with and without filters. (a) Three photography filters (BPM1, BPM2, and R3020) and three neutral density filters (ND 0.1, ND 0.2, and ND 0.3) were used with the artificial eye. The straight lines correspond to the linear fit performed. (b) Ocular transmittance index (OTI) as a function of the transmittance measured in the filters. OTI values are the slopes of the straight lines fitted to the data showed in a.

From the curve fitted to the data plotted in Fig. $6 \mathbf{b}$ the following expression can be deduced:

$$
\mathrm{OTI}=84.5 T^{2}
$$

Using Eq. (1), it is possible to estimate the direct transmittance of ocular media $T$ from the OTI value.

Figure 7 shows the effect of reflectance of the fundus on the measurement of OTI. The reflectance measured with the spectroradiometer at $780 \mathrm{~nm}$ in the black and white cardboard was $7.9 \%$ and $78.6 \%$, respectively. The results presented in Fig. 7 a show 


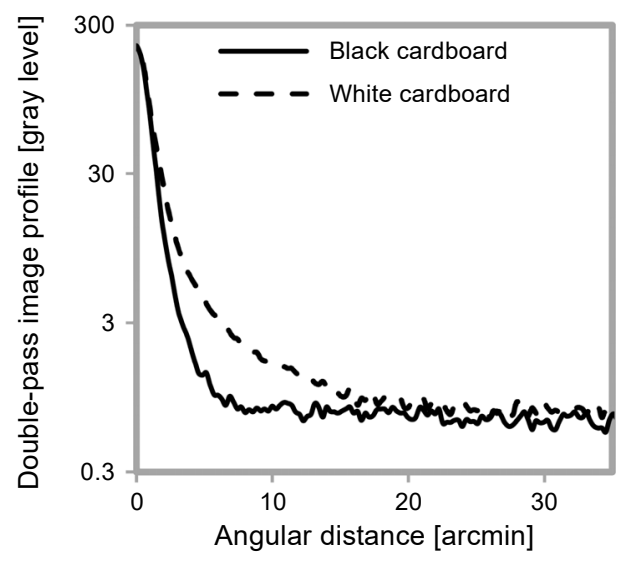

a

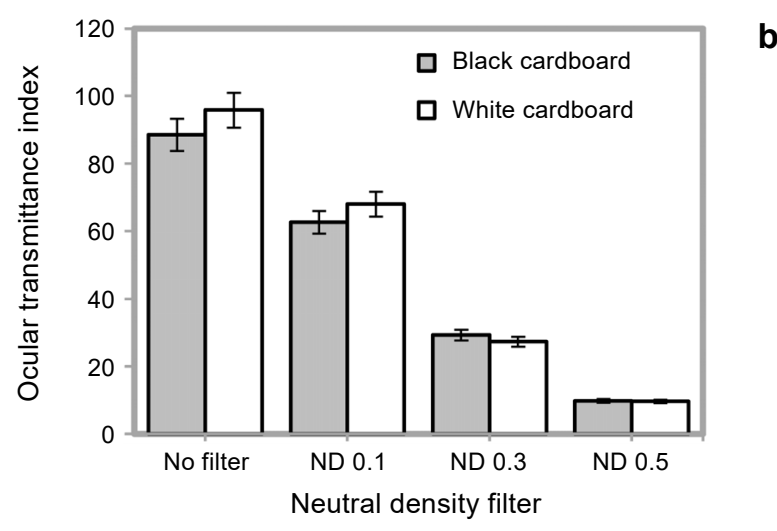

Fig. 7. Analysis of the effect of reflectance on the measurement of the ocular transmittance index (OTI). (a) Double-pass (DP) image profile in the logarithmic scale obtained for two different cardboards used as artificial retinas (black and white) measured at the same power of the laser. (b) OTI values measured in the artificial eye with two different fundi (black and white cardboards) without filter (no filter) and with three neutral filters (ND 0.1, ND 0.3, and ND 0.5).

that although very different profiles of DP images were obtained in each condition, there are no significant changes in the peripheral zone of the curves (note that the vertical axis is in the logarithmic scale). Figure $7 \mathbf{b}$ shows OTI values obtained in the artificial eye with and without filters, with both black and white cardboards acting as a retina. No statistically significant differences were found for the two cardboards for the artificial eye without filter $(p=0.1041)$ and for the artificial eye with filters $(p=0.2730$ (ND 0.1), $p=0.0757$ (ND 0.3), and $p=0.9097$ (ND 0.5)).

Figure 8 a shows the DP image profiles determined in five subjects with similar OTI values. Figure $8 \mathbf{b}$ shows the box plots corresponding to the gray level of the ROI for five subjects. No statistically significant differences were found for the gray level of the DP image in the ROI evaluated for five subjects $(p>0.05)$.

The OTI values for the artificial eye as a function of defocus are shown in Fig. 9. To determine the influence of the defocus on the OTI, Mann-Whitney tests were performed 


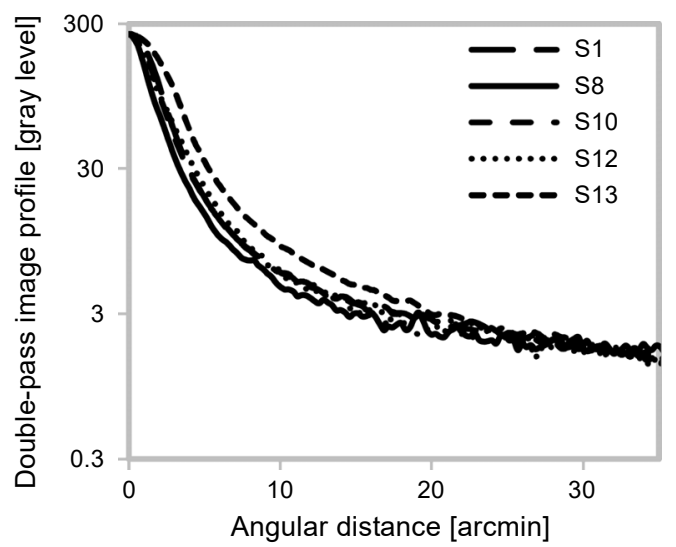

a

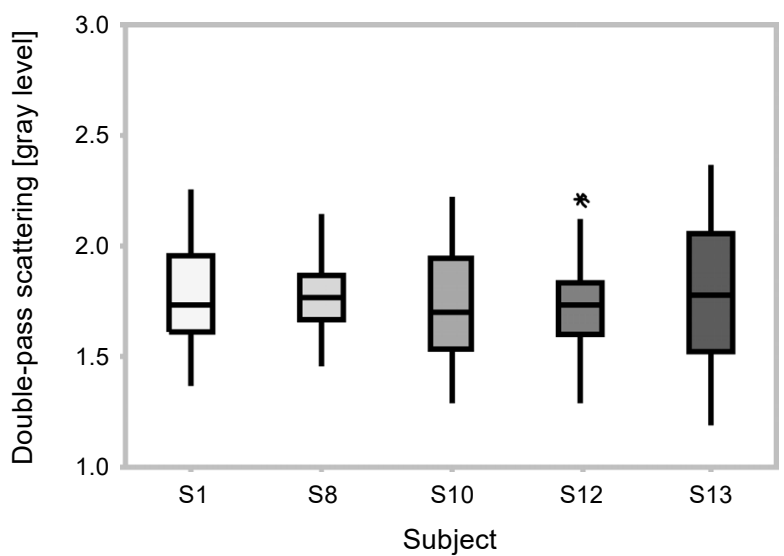

b

Fig. 8. Double-pass (DP) images profiles in the logarithmic scale. (a) Five DP images were determined at the same laser power in five subjects. (b) Box plots corresponding to the gray level of the region of interest defined to compute the double-pass scattering for five subjects.

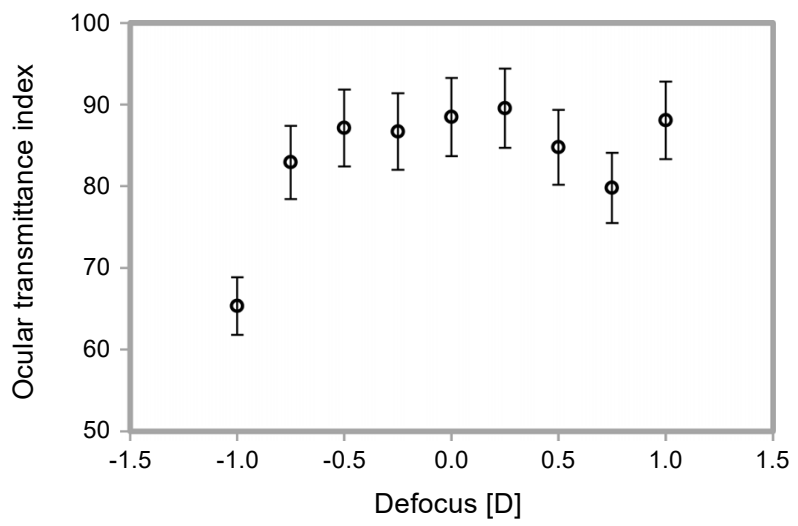

Fig. 9. Ocular transmittance index (OTI) as a function of induced defocus. OTI value was measured in the artificial eye at the best focus position and with induced defocus from -1.0 to $+1.0 \mathrm{D}$ in $0.25 \mathrm{D}$ steps. 
to compare the condition of best focus with each condition of induces defocus. No statistically significant differences were found for the OTI value measured in the artificial eye with induced defocus between -0.75 and $+0.50 \mathrm{D}(p>0.05)$. Only for a defocus of -1.0 and $+0.75 \mathrm{D}$ statistically significant difference was found $(p<0.05)$.

In Fig. 10 are presented DPS values as a function of the irradiance of the laser in the pupil plane for subjects of group A, and the straight lines fitted to the data. All the data show a good linear fit, with a coefficient of determination always greater than 0.82 .
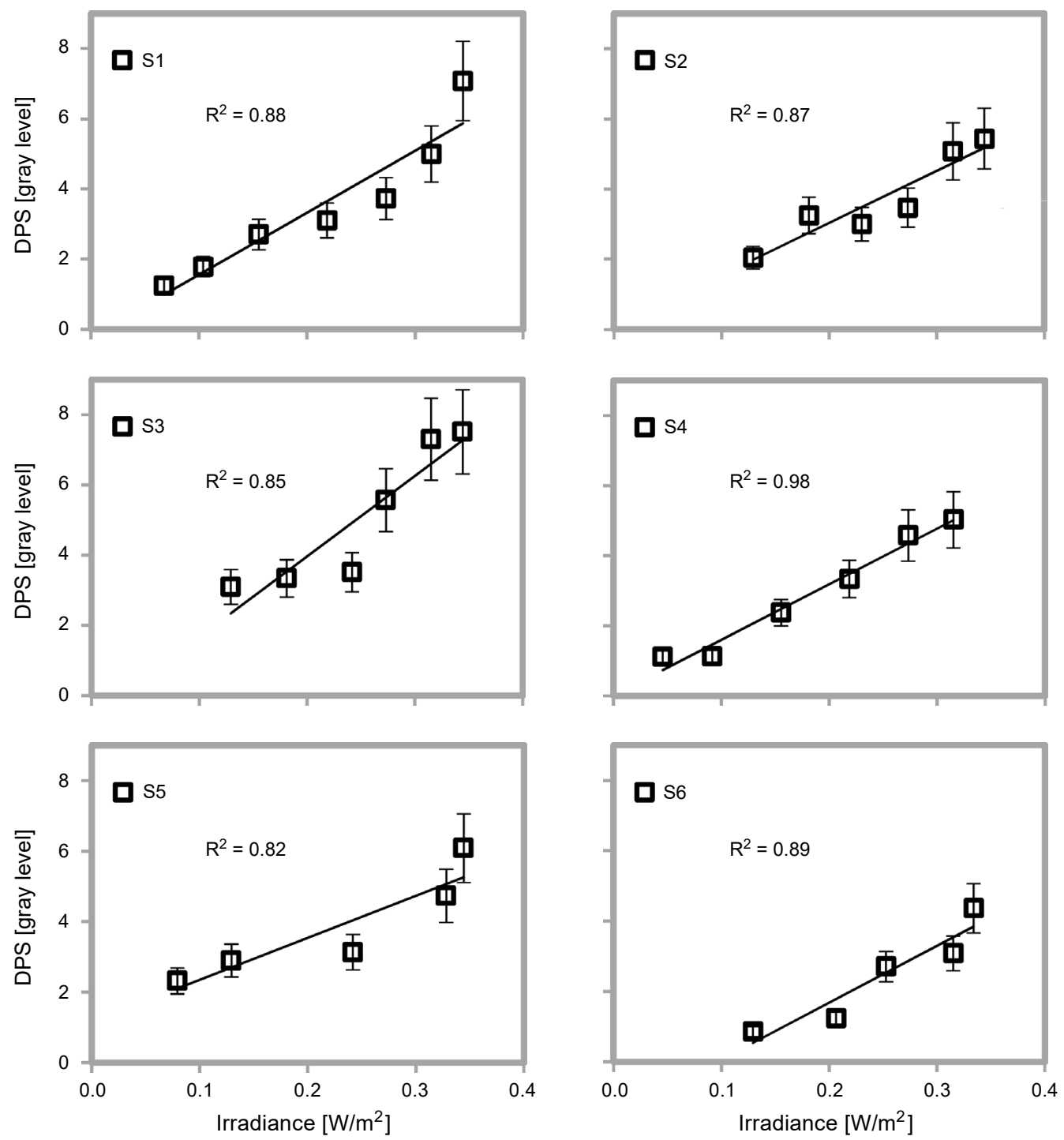

Fig. 10. Double-pass scattering (DPS) expressed in terms of the gray level as a function of the irradiance of the laser diode in the pupil plane. The straight line fitted and the coefficient of determination are shown for each subject. The error bars represent the standard deviation from the mean. 

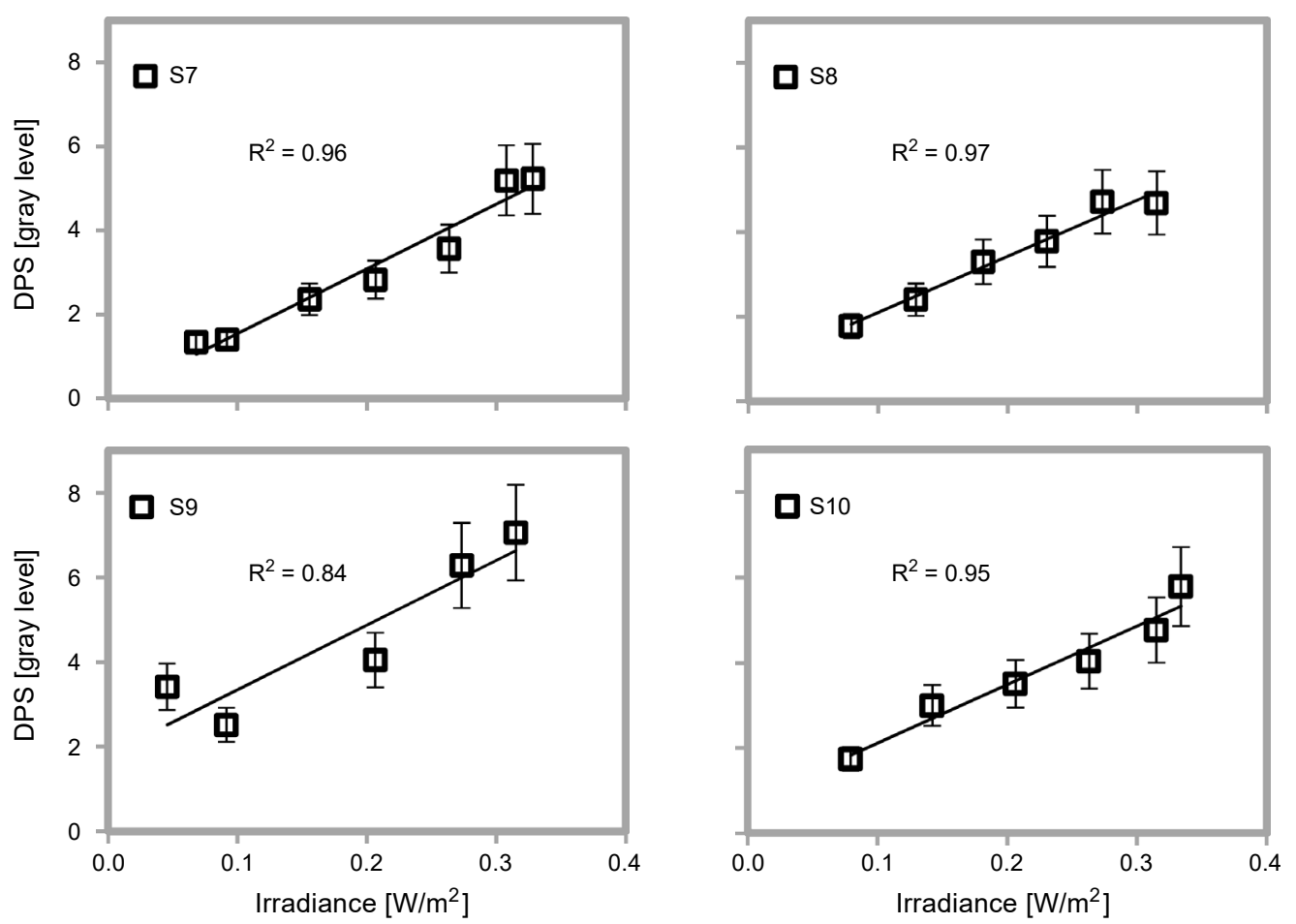

Fig. 10. Continued.

Table 2 presents the OTI values determined in each subject and the direct transmittance value computed from Eq. (1).

The values obtained of direct transmittance for subjects of group A ranged from $37.5 \%$ to $52.1 \%$, with a mean value of $42.95 \pm 3.97 \%$ (mean $\pm \mathrm{SD})$. The mean value

$\mathrm{T}$ a b 1 e 2. Ocular transmittance index (OTI) and direct transmittance of the eye for subjects of group A.

\begin{tabular}{lll}
\hline Subject (iris color) & OTI & $\begin{array}{l}\text { Direct transmittance } \\
\text { of the eye [\%] }\end{array}$ \\
\hline S1 (dark) & 17.7 & $45.7 \pm 2.5$ \\
S2 (dark) & 14.9 & $41.9 \pm 2.3$ \\
S3 (dark) & 23.0 & $52.1 \pm 2.8$ \\
S4 (dark) & 15.9 & $43.3 \pm 2.3$ \\
S5 (dark) & 11.9 & $37.5 \pm 2.0$ \\
S6 (dark) & 16.2 & $43.7 \pm 2.4$ \\
S7 (dark) & 15.4 & $42.7 \pm 2.3$ \\
S8 (light) & 13.3 & $39.7 \pm 2.1$ \\
S9 (light) & 15.3 & $42.5 \pm 2.3$ \\
S10 (light) & 13.7 & $40.3 \pm 2.2$ \\
\hline
\end{tabular}

Pigmentation (iris color) is shown in parentheses. 
for subjects with light eyes was $40.84 \pm 1.52 \%$ (mean $\pm \mathrm{SD}$ ), whereas for subjects with dark eyes, the mean was $43.86 \pm 4.43 \%$ (mean $\pm \mathrm{SD}$ ). No significant differences were found in the transmittance for the subjects with light eyes and for subjects with dark eyes $(p=0.154)$.
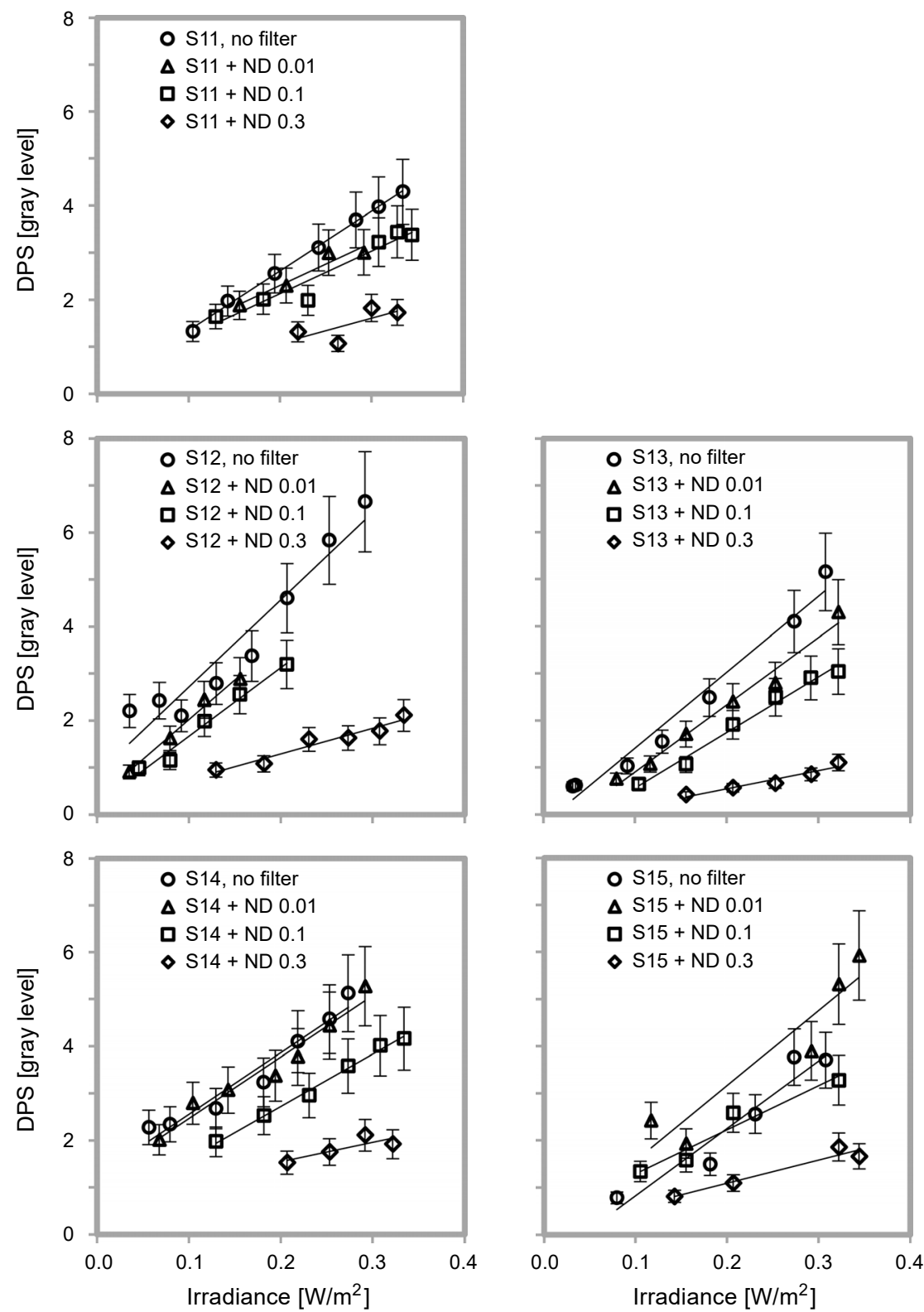

Fig. 11. Double-pass scattering (DPS) expressed in terms of the gray level as a function of the irradiance of the laser obtained in the eye of five subjects with and without filters. The straight lines correspond to the linear fit performed to the data. The error bars represent the standard deviation from the mean. 
$\mathrm{T}$ a b l e 3. Ocular transmittance index (OTI) and direct transmittance of the eye for each subject of group B with and without filters.

\begin{tabular}{lrl}
\hline Condition & OTI & $\begin{array}{l}\text { Direct transmittance } \\
\text { of the eye [\%] }\end{array}$ \\
\hline S11 without filter & 12.7 & $38.7 \pm 2.1$ \\
S11 with ND 0.01 & 9.1 & $32.8 \pm 1.8$ \\
S11 with ND 0.1 & 9.1 & $32.7 \pm 1.8$ \\
S11 with ND 0.3 & 5.4 & $25.2 \pm 1.4$ \\
\hline S12 without filter & 18.6 & $46.9 \pm 2.5$ \\
S12 with ND 0.01 & 16.9 & $44.7 \pm 2.4$ \\
S12 with ND 0.1 & 14.6 & $41.6 \pm 2.2$ \\
S12 with ND 0.3 & 5.5 & $25.4 \pm 1.4$ \\
S13 without filter & 16.2 & $43.7 \pm 2.4$ \\
S13 with ND 0.01 & 14.3 & $41.1 \pm 2.2$ \\
S13 with ND 0.1 & 11.8 & $37.4 \pm 2.0$ \\
S13 with ND 0.3 & 3.9 & $21.4 \pm 1.2$ \\
\hline S14 without filter & 13.1 & $39.4 \pm 2.1$ \\
S14 with ND 0.01 & 13.1 & $39.3 \pm 2.1$ \\
S14 with ND 0.1 & 11.1 & $36.2 \pm 2.0$ \\
S14 with ND 0.3 & 4.9 & $22.2 \pm 1.2$ \\
\hline S15 without filter & 14.3 & $41.1 \pm 2.2$ \\
S15 with ND 0.01 & 15.9 & $43.4 \pm 2.3$ \\
S15 with ND 0.1 & 9.4 & $33.3 \pm 1.8$ \\
S15 with ND 0.3 & 4.9 & $24.1 \pm 1.3$ \\
\hline
\end{tabular}

OTI - ocular transmittance index, ND - neutral density filter.

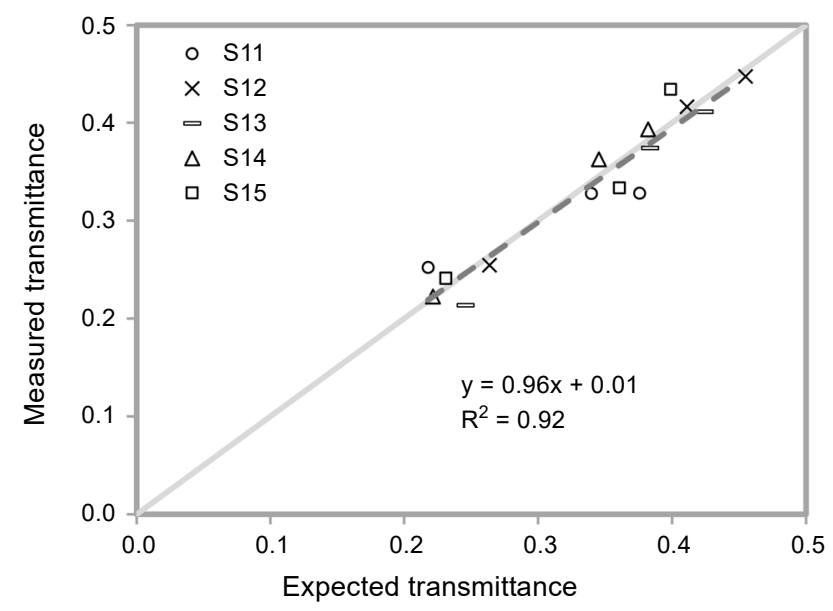

Fig. 12. Direct transmittance measured in the subjects of group B with filters as a function of the expected transmittance value. The straight line fitted to the data (dashed line) along with its equation and the coefficient of determination are shown. The line of equality $(y=x)$ is shown for comparison purposes (solid line). 
Figure 11 shows the DPS values for four conditions evaluated (eye without a filter, eye + ND 0.01, eye + ND 0.1, and eye + ND 0.3) for five subjects of the group B and the fit performed to the data. The OTI values and the direct transmittances calculated from Eq. (1) are shown in Table 3.

From the transmittance values measured in the filters (Table 1) and the estimated transmittance values in the subjects of group B (Table 3, condition "without filter") we have calculated the expected transmittance for the measurements made on the eyes with filters. For example, the subject S12 has a transmittance of $46.9 \%$, therefore when measuring the eye of this subject with the filter ND $0.1(T=87.7 \%)$ the expected transmittance was calculated as the product of the transmittance of S12 by the transmittance of the filter ND 0.1 , obtaining $41.1 \%$. Figure 12 shows the transmittance values measured in the subjects of group B with three neutral filters (ND 0.01, ND 0.1, and ND 0.3), as a function of the expected transmittance. The high correlation $(r=0.959, p<0.0001)$ showed in Fig. 12 was expected and adds consistency to our results.

\section{Discussion}

In this article, we have proposed a methodology to measure the in vivo direct transmittance of the whole eye, that is, including the retina. BOETTNER reported $54 \%$ for direct transmittance at $780 \mathrm{~nm}$ in eyes corresponding to a range of age similar to that used in our work [ $\underline{3}]$. This value corresponds to the mean transmittance of pre-retinal media (cornea, aqueous humor, lens, and vitreous) measured in a sample of enucleated eyes with clear ocular media. Moreover, BOETTNER showed a transmittance measurement of the rhesus monkey retina, considered anatomically similar to that of the human eye, which was $80 \%$ for a wavelength of $800 \mathrm{~nm}$. Taking these results into account, the expected value for direct transmittance of the ocular media plus the retina can be calculated by multiplying the transmittance of the pre-retinal media by the transmittance of the retina, which results in a total transmittance of $43.2 \%$. Our experimental results, based on double-pass (DP) measurements that record the image reflected in the inner layers of the retina, provide a mean value for the direct transmittance at $780 \mathrm{~nm}$ of the eye including the retina of $42.95 \%$, which agrees with the expected value for the transmittance at that wavelength. A single-sample $t$-test was used to compare the mean direct transmittance of group A (Table 2) with the expected value for the mean transmittance based on the results obtained by BOETTNER and no significant difference was found $(p=0.848)$.

Christaras et al. [22] have reported differences in a small sample in the straylight measured in subjects with light eyes and dark eyes. Christaras's measurements show that diffused light from the fundus contributes significantly to the total straylight for wavelengths longer than $600 \mathrm{~nm}$, especially in the measurements made for small-angles of the PSF (30 minutes of arc). In our study, no significant differences were found between the transmittances measured in subjects with different pigmentation.

The DP technique provides combined information of both aberrations and ocular scatter. Recently, ZHAO et al. obtained a significant increase in the optical quality of the DP PSF by correcting the ocular aberrations through an adaptive optics system up to the 
eighth Zernike order [299]. When comparing the retinal images with defocus and astigmatism corrected with the images achieved with aberrations corrected up to the eighth Zernike order, an obvious reduction in the spatial extent of the DP PSF and an increase in the contrast were observed, suggesting that higher-order aberrations degraded the DP PSF. As with other optical quality measurements derived from the DP method [ $\underline{30}$ ], our measurement of transmittance was affected by uncorrected low -order aberrations (defocus and astigmatism). Uncorrected refractive errors over \pm 0.75 diopters induce artifacts on the determination of the ocular transmittance index (OTI) (Fig. 9). This indicates that refractive errors need to be well corrected during the DP data collection to provide accurate values of OTI. In our measurements, the refractive errors of the subjects were corrected with the spherical equivalent by means of the Badal system, therefore, in some subjects, there could be a little effect of uncorrected astigmatism that adds error to the measure.

The measurement of OTI took a few minutes, therefore, eye movements were inevitable. This would cause the laser to strike a different area of the retina with a slightly different reflectance, however, those variations did not produce changes in the measured OTI. In that sense, the behavior of the eye was similar to that presented by the artificial eye (Fig. 7). Comparing Figs. 7a and 8a, the DP PSF in real eyes drops off much slower than the DP PSF in the artificial eye and does not reach a horizontal asymptote. The artificial eye used in our work is a very simplified eye model, especially with regard to the fundus of the eye. Although the cardboard used as a fundus in the artificial eye has a reflectance value comparable to that obtained in real eyes $(7.9 \%$ for black cardboard), it represents a single scattering surface. The ocular fundus consists of different layers, some very vascularized as the choroid where light, especially far-red and near-infrared penetrate and diffuse [31]. The light coming from those layers can contribute to the light scattered in the DP image and may explain the differences observed between the artificial and the real eye. In general, in the most eccentric areas (30-35 minutes of arc) there was an overestimation of the DP PSF measured in the artificial eye, while in the areas closest to the center (20-25 minutes of arc) an underestimation of the DP PSF of the artificial eye was observed. However, these differences were reduced by averaging of the gray level in the region of interest for the analysis, as has been proposed in our work.

According to Fig. 12, the technique described in this work can be used for a certain range. Because low values of transmittance have a significant error, the method would not be suitable for eyes with significant media opacity. The low levels of energy that reach the sensor in the region of interest evaluated limits the scope of the proposed method since in this zone there is a low signal-to-noise ratio, due to the low dynamic range of the 8 bits CCD sensor commonly used in DP instruments. This might become a critical issue when the method is applied to measure the transmittance of blue or UV light, maintaining ocular energy exposure under guidelines. A 12 bits camera would be of assistance to enhance the dynamic range. However, since we are only interested in the energy reaching the peripheral zone and not in the whole PSF, more sensitive single-pixel 
sensors could be used instead of a CCD to increase the dynamic range and record the lower energy levels.

Another limitation of the proposed method is that the transmittance was measured at a single wavelength and does not cover a spectral range, while in the most cited works, a spectral transmittance curve for the ocular media was measured [2- $\underline{5}]$. However, it would be possible to determine the transmittance for other wavelengths using the same method described in our work, considering the modification in the sensor previously mentioned. In addition, a double-pass system with a polychromatic source has been developed in recent years [32] and the spectral transmittance of the human eye could be also measured based on the same configuration and the procedure described here. The spectral transmittance can be useful for specific applications such as in the study of intrinsically photosensitive retinal ganglion cells using a five-primary photostimulator, where it is necessary to characterize the individual differences in pre-receptoral filtering in each subject $[\underline{33}, \underline{34}]$.

Finally, the method presented in this paper allows a quick and non-invasive measurement of transmittance, which can also be automated and does not require the instillation of mydriatic or cycloplegic agents. It is also independent of the active participation of the subject and does not require performing difficult tasks, as occurs in psychophysical measures.

\section{Conclusion}

We have developed a procedure to determine the transmittance of the human eye in vivo for a wavelength of $780 \mathrm{~nm}$ using the double-pass method, commonly used for the determination of the optical quality of an eye. The process requires taking double-pass images with different laser beam intensities and determining the slope of the straight line fitted to the obtained data. From this value and using a calibration equation we have described here, the direct transmittance of the eye have been obtained.

Acknowledgements - We thank Jose Barraza, Ph.D., UNT-CONICET, Barbara Silva, Ph.D., UNT-CONICET, and Raquel Castillo, UNT-CONICET, for the assistance and for comments that greatly improved the manuscript.

\section{References}

[1] Le Grand Y., Light, Colour and Vision, Second English Edition, Chapman and Hall, London 1968.

[2] Boettner E.A., Wolter J.R., Transmission of the ocular media, Investigative Ophthalmology and Visual Science 1(6), 1962, pp. 776-783.

[3] Boettner E.A., Spectral transmission of the eye, Final contract report AF41(609)-2966, University of Michigan, Ann Arbor MI, July 1967. [Available from NTIS as AD 663246].

[4] GeERAETS W.J., BERRY E.R., Ocular spectral characteristics as related to hazards from lasers and other light sources, American Journal of Ophthalmology 66(1), 1968, pp. 15-20, DOI: 10.1016/0002-9394 (68)91780-7.

[5] Alpern M., Thompson S., Lee M.S., Spectral transmittance of visible light by the living human eye, Journal of the Optical Society of America 55(6), 1965, pp. 723-727, DOI: 10.1364/JOSA.55.000723. 
[6] Dillon J., Zheng L., Merriam J.C., Gaillard E.R., Transmission spectra of light to the mammalian retina, Photochemistry and Photobiology 71(2), 2000, pp. 225-229, DOI: $\underline{10.1562 / 0031-8655(2000)}$ 0710225TSOLTT2.0.CO2.

[7] Norren D.V., Vos J.J., Spectral transmission of the human ocular media, Vision Research 14(11), 1974, pp. 1237-1244, DOI: 10.1016/0042-6989(74)90222-3.

[8] VAn de KraAts J., VAn NorRen D., Optical density of the aging human ocular media in the visible and the UV, Journal of the Optical Society of America A 24(7), 2007, pp. 1842-1857, DOI: 10.1364/ JOSAA.24.001842.

[9] Lund D.J., Marshall J., Mellerio J., Okuno T., Schulmeister K., Sliney D., et al., A computerized approach to transmission and absorption characteristics of the human eye, CIE 203:2012, 2012, International Commission on Illumination.

[10] Santamaría J., Artal P., Bescós J., Determination of the point-spread function of human eyes using a hybrid optical-digital method, Journal of the Optical Society of America A 4(6), 1987, pp. 1109-1114, DOI: $10.1364 /$ JOSAA.4.001109.

[11] Artal P., Marcos S., Navarro R., Williams D.R., Odd aberrations and double-pass measurements of retinal image quality, Journal of the Optical Society of America A 12(2), 1995, pp. 195-201, DOI: 10.1364/JOSAA.12.000195.

[12] Guirao A., LóPez-Gil N., Artal P., Double-pass measurements of retinal image quality: a review of the theory, limitations, and results, Vision Science and its Applications, OSA Technical Digest, Optical Society of America, 2000, paper NW4, DOI: 10.1364/VSIA.2000.NW4.

[13] López-Gil N., ARTAL P., Comparison of double-pass estimates of the retinal image quality obtained with green and near-infrared light, Journal of the Optical Society of America A 14(5), 1997, pp. 961 -971, DOI: 10.1364/JOSAA.14.000961.

[14] Ginis H.S., Perez G.M., Bueno J.M., Pennos A., Artal P., Wavelength dependence of the ocular straylight, Investigative Ophthalmology and Visual Science 54(5), 2013, pp. 3702-3708, DOI: $10.1167 /$ iovs.13-11697.

[15] Van Den Berg T.J., Franssen L., Coppens J.E., Straylight in the human eye: testing objectivity and optical character of the psychophysical measurement, Ophthalmic and Physiological Optics 29(3), 2009, pp. 345-350, DOI: 10.1111/j.1475-1313.2009.00638.x.

[16] Van Den Berg T.J., Analysis of intraocular straylight, especially in relation to age, Optometry and Vision Science 72(2), 1995, pp. 52-59.

[17] Franssen L., Tabernero J., Coppens J.E., van den Berg T.J., Pupil size and retinal straylight in the normal eye, Investigative Ophthalmology and Visual Science 48(5), 2007, pp. 2375-2382, DOI: 10.1167/iovs.06-0759.

[18] Van den Berg T.J., Franssen L., Kruijt B., Coppens J.E., History of ocular straylight measurement: A review, Zeitschrift für Medizinische Physik 23(1), 2013, pp. 6-20, DOI: 10.1016/j.zemedi.2012. $\underline{10.009}$.

[19] Artal P., Benito A., Pérez G.M., Alcón E., de Casas A., Pujol J., Marín J.M., An objective scatter index based on double-pass retinal images of a point source to classify cataracts, PLoS ONE 6(2), 2011, article e16823, DOI: 10.1371/journal.pone.0016823.

[20] Vilaseca M., Romero M.J., Arjona M., Luque S.O., Ondategui J.C., Salvador A., Güell J.L., Artal P., Pujol J., Grading nuclear, cortical and posterior subcapsular cataracts using an objective scatter index measured with a double-pass system, British Journal of Ophthalmology 96(9), 2012, pp. 1204-1210, DOI: 10.1136/bjophthalmol-2011-301055.

[21] Paz Filgueira C., SÁnchez R.F., Issolio L.A., Colombo E.M., Straylight and visual quality on early nuclear and posterior subcapsular cataracts, Current Eye Research 41(9), 2016, pp. 1209-1215, DOI: $10.3109 / 02713683.2015 .1101139$.

[22] Christaras D., Ginis H., ARTAl P., Spatial properties of fundus reflectance and red-green relative spectral sensitivity, Journal of the Optical Society of America A 32(9), 2015, pp. 1723-1728, DOI: 10.1364/JOSAA.32.001723.

[23] Safety of Laser Products, IEC 60825-1 Standard, 2007. 
[24] Díaz-Doutón F., Benito A., Pujol J., Arjona M., Güell J.L., Artal P., Comparison of the retinal image quality with a Hartmann-Shack wavefront sensor and a double-pass instrument, Investigative Ophthalmology and Visual Science 47(4), 2006, pp. 1710-1716, DOI: 10.1167/iovs.05-1049.

[25] Hofer H., Artal P., Singer B., Aragón J.L., Williams D.R., Dynamics of the eye's wave aberration, Journal of the Optical Society of America A 18(3), 2001, pp. 497-506, DOI: 10.1364/JOSAA.18.000497.

[26] Barrionuevo P.A., Colombo E.M., Vilaseca M., Pujol J., Issolio L.A., Comparison between an objective and a psychophysical method for the evaluation of intraocular light scattering, Journal of the Optical Society of America A 29(7), 2012, pp. 1293-1299, DOI: 10.1364/JOSAA.29.001293.

[27] De Wit G.C., Franssen L., Coppens J.E., van Den Berg T.J., Simulating the straylight effects of cataracts, Journal of Cataract and Refractive Surgery 32(2), 2006, pp. 294-300, DOI: 10.1016/ j.jcrs.2006.01.048.

[28] Logean E., Dalimier E., Dainty C., Measured double-pass intensity point-spread function after adaptive optics correction of ocular aberrations, Optics Express 16(22), 2008, pp. 17348-17357, DOI: $10.1364 / \mathrm{OE} .16 .017348$.

[29] Zhao J., Xiao F., Kang J., Zhao H., Dai Y., Zhang Y., Quantifying intraocular scatter with near diffraction-limited double-pass point spread function, Biomedical Optics Express 7(11), 2016, pp. 4595-4604, DOI: 10.1364/BOE.7.004595.

[30] Martínez-Roda J.A., Vilaseca M., Ondategui J.C., Giner A., Burgos F.J., Cardona G., Pujol J., Optical quality and intraocular scattering in a healthy young population, Clinical and Experimental Optometry 94(2), 2011, pp. 223-229, DOI: 10.1111/j.1444-0938.2010.00535.x.

[31] Berendschot T.T., DeLint P.J., van Norren D., Fundus reflectance-historical and present ideas, Progress in Retinal and Eye Research 22(2), 2003, pp. 171-200, DOI: 10.1016/S1350-9462(02)00060-5.

[32] Ginis H., Pérez G.M., Bueno J.M., Artal P., The wide-angle point spread function of the human eye reconstructed by a new optical method, Journal of Vision 12(3), 2012, article 20, DOI: 10.1167/ 12.3.20.

[33] Cao D., Nicandro N., Barrionuevo P.A., A five-primary photostimulator suitable for studying intrinsically photosensitive retinal ganglion cell functions in humans, Journal of Vision 15(1), 2015, article 27, DOI: $10.1167 / 15.1 .27$.

[34] BARrionuevo P.A., CAO D., Luminance and chromatic signals interact differently with melanopsin activation to control the pupil light response, Journal of Vision 16(11), 2016, article 29, DOI: 10.1167/16.11.29. 